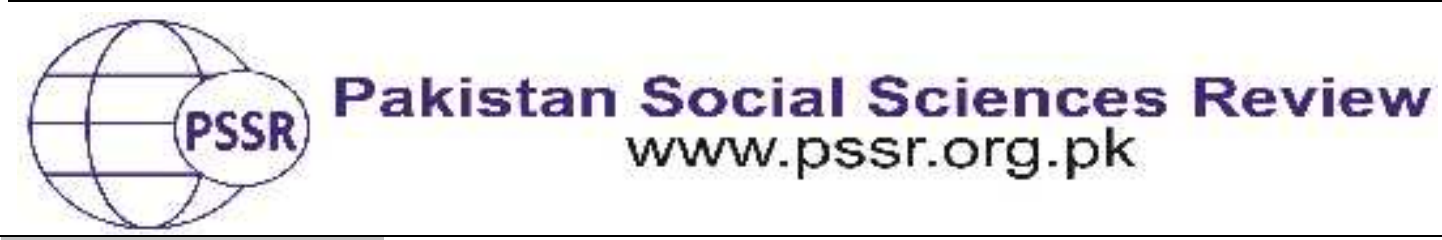

RESEARCH PAPER

\title{
Standardization of Adapted Picture Exchange Communication System (PECS) For Children with ASD in Pakistan
}

\author{
Farah Rahman ${ }^{1}$ Dr. Almas Ikram kayani ${ }^{2}$
}

1. Ph. D Scholar, Department of Education \& FSS , Pir Mehr Ali Shah Arid Agriculture University, Rawalpindi, Punjab, Pakistan

2. Associate Professor, Department of Education\& FSS, Pir Mehr Ali Shah Arid Agriculture University, Rawalpindi, Punjab, Pakistan

\begin{tabular}{|c|c|}
\hline PAPER INFO & ABSTRACT \\
\hline Received: & The current experimental research aimed to standardize the \\
\hline January 21, 2020 & adapted version of PECS for autistic children in Pakistan. The \\
\hline Accepted: & Adapted PECS was standardized by an experimenta \\
\hline 20 & hildren \\
\hline ), 2020 & of \\
\hline Key & for \\
\hline Cul & PECS in Pakistan. Primary data was collected \\
\hline Adaptation, & by $\mathrm{c}$ \\
\hline $\begin{array}{l}\text { nce Based } \\
\text { ces. }\end{array}$ & stic \\
\hline nal & celiability and validity. An \\
\hline ication, & adapted version of PECS justifies the criteria of a suitable sample \\
\hline & size, concurrent validity, predictive validity, measures of central \\
\hline Pro & tendency and variability, test-retest reliability, and \\
\hline Corresponding & y. The data analysis results illustrated that adapted \\
\hline & alid and reliable tool to be managed in \\
\hline 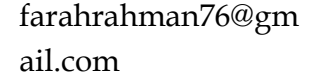 & \\
\hline
\end{tabular}

\section{Introduction}

Picture exchange communication system (PECS) is a renowned, effective intrusion system, which is considered to progress functional communication abilities (Bondy\& Frost, 2001). It is a kind of alternative and augmentative communication (AAC) approach designed by pyramid educational consultants, and it is frequently used to progress functional skills in autistics. It has been used for many children, from 2 to 16 years age group, who indicate many communications, reasoning, and other bodily deficiencies such as palsy of any brain part, visual impairment, and hearing impairment. In it, methodical training of functional communication skills involved during the phases (Bondy\& Frost, 2001). Researches show that it is 
operative predominantly in making requests. It hypothetically provides a bridge to develop speech and language skills. Andy and Lori have developed it, and in the start, mostly young children (age of pre-schooler) with autism spectrum disorder (ASD) used it and other children with speech and language disorders, who shows no purposeful and effective communication also use this modality (Frost \& Bondy, 2012). However, in the picture exchange communication system, there is a huge \& large PECS book comprising plenteous picture cards which would have to be along with the user where ever he/she wishes to communicate. Replies and reactions would be limited to the existing pictures. A quantifiable and constituent exploration of PECS, conducted by (Tincani \& Devis, 2011) in single-participant studies.

Kumpfer et al. (2002), has specified the sense of ethnic based alteration, is to adapt the tool/system or program according to the philosophy and principals of a particular area.

Alteration of any program or intervention according to culture into Evidence Based Practices (EBP) that contains the re-check and change of the structure of intervention to fit it more appropriately according to the necessities of a specific culture . The alteration of any evidence-based intervention or tool is a difficult task, and it also needs methodical and vigilant organization and preparation to keep the protocol, psychometrics, and collective strength through validation intended for the designated people (Cassepp, Borges, Balbinotti, \&Teodoro, 2010). Its process must be provided, evidence of significant consistency of material and the capability through validation of the adapted intervention tool (International Test Commission [ITC], 2010). It is the matter of primary significance that altered instrument according to cultural norms, and values must have the characteristics to use it in other societal context (Beaton et al., 2000; Sireci et al., 2006).

In the alteration procedure, important, suitable language, equivalence and psychometric calculation of the properties should be measured among the new altered and the old original tool (ITC, 2010). The initial step of the alteration procedure is to translate the instrument (adapted word list of PECS) according to the inherent language. During the progression of alteration of an intrusion, social norms and values, features related to its linguistics and context should be measured regarding its translation.

The current research aimed to highlight the standardization of adapted edition of this tool for children with autistic in Pakistan. The objective of the study was:

1. To assess the reliability and validity of adapted PECS for autistic children of Pakistan.

The hypothesis was formulated for the study that, there is no significant difference between the reliability of pre-testing and post- testing. 


\section{Material and Method}

The current study completed into two steps. In first step reliability of adapted picture exchange communication was evaluated and in the second step adapted PECS was validated.

Psychometric calculation and valuation of intrusion tools are obligatory to implement the tool in any practical field. And psychometric strength of every test may deceit the stability, reliability and strength. The stability and consistency of any test is apprehensive through firmness of the results of tests. By altering all those procedures which are used for organizing the reliability of original PECS, stability and consistency of altered PECS was recognized. The psychometric competence of a gadget includes the evaluation of its reliability and item statistics (e.g., item-total correlations and variances or standard deviations) and validity. Psychometric properties of both original and altered PECS designed for autistic children were obtained through the key samples particularly by the application of alpha reliability coefficient, inter-scale correlation and items-total correlations on all. Psychometric calculation and expansion of social norms are involved for the consistency of every test. In the last stage of the final trial, the consistency of altered PECS accomplished, and it has concluded by the post-testing with the altered PECS on the sample of seventy (70) normal and thirty (30) autistic children to validate the tool.

As cited in Sireci et al. (2006) the typical measures were done for the validation of tailored version in equally the ITC test alteration guiding principle as well as the principles defined for instructive and measurement of psychometric properties (American Educational Research) association, American psychological association and National council on measurement in education. A detailed as well as systematic calculation of entirely altered in other words tailored pictures was accepted, through statistics techniques. The purpose of these techniques was to improve the reliability of a tool.

\section{First step: Reliability of adapted picture exchange communication system}

The reliability of modified and altered PECS was measured by the different types of reliability. The results for all the reliability types indicates that Inter-rater reliability of adapted PECS is greater than 0.8, and adapted PECS has a high test retest reliability, the mean reliability coefficients of altered PECS are higher than original PECS for the sample of Pakistan; proving to be a reliable educational tool.

\section{Second step: Validation of altered picture exchange communication system}

Additionally altered PECS has also met all validity types except convergent validity. Altered PECS met all criteria of content validity including concurrent validity, predictive/discriminant, cross-cultural validity and construct validity. 
Experimental research design was deployed. The current study is a statistical study and of quantitative nature. Sample statistics were used to normalize the population parameters. Non-probability and probability techniques were organized to curtail the biasness.

\section{Sample}

The sample of 30 autistic students were drawn through convenience sampling from 7 special education institutes of Rawalpindi/Islamabad and 70 normal students studying in the primary section of 2 model colleges for girls and boys of Islamabad ( 35 girls and 35 boys), for post testing, selected through simple random sampling.

\section{Instrument}

Three thousand nine hundred and nine (3909) picture cards of the altered and adapted PECS in the digital form (soft form) and self-developed checklist for the marking of responses of autistic children and normal children by the committee members.

\section{Procedure}

Statistics was used for data analysis. To conclude the outcomes, coding of responses is of chief importance in computable and measureable research. The questions and answers of the participants were entered and coded in the excel sheets.

The reliability of altered PECS was evaluated by internal consistency, interrater, and test-retest reliability. The results for all these reliability types indicates that Inter-rater reliability of altered PECS is greater than 0.8 , and it has a high test retest reliability, the mean reliability coefficients of adapted/altered PECS are higher than original PECS for the sample of Pakistan; proving to be a reliable educational tool; results are elaborated statistically.

\section{Results and Discussion}

The highest apprehension of all students who compile the educational data is validity. By connecting all items with the objectives and hypothesis of the study, content validity of this tool was determined. Following statistical analysis was used to standardize the original and altered PECS.
a. Alpha reliability coefficient
b. Inter-scale correlation of the categories of PECS
c. Items- total correlations. 
Table 1

Reliability coefficient centered on autistic student's recognition for 43 categories of altered PECS during post-testing $(\mathrm{N}=30)$

\begin{tabular}{ccc}
\hline $\begin{array}{c}\text { Overall Reliability (Post testing } \\
\text { Autistic Data) }\end{array}$ & No. of Items & Alpha Coefficient \\
\hline & 43 & 0.868 \\
\hline
\end{tabular}

Table 1 showed total reliability coefficient based on autistic student's recognition for 43 categories of altered and modified PECS during post-testing phase. The total alpha coefficient for 43 items was in good range as its value 0.868 was greater than 0.8 . Greater than 0.8 value of alpha concluded relatively higher internal consistency among 43 sub-categories of altered PECS.

Table 2

Reliability coefficient centred on normal student's recognition for 43 categories of altered PECS during post-testing $(\mathrm{N}=70)$

\begin{tabular}{ccc}
\hline $\begin{array}{c}\text { Overall Reliability (Post testing } \\
\text { Normal Data) }\end{array}$ & No. of Items & Alpha Coefficient \\
\hline & 43 & 0.8369 \\
\hline
\end{tabular}

Table 2 demonstrates total reliability coefficient centred on normal student's recognition for 43 categories of altered PECS during post-testing phase. The overall alpha coefficient for 43 items was in good range as its value 0.8369 was greater than 0.8 . This 0.8 value of alpha determined comparatively higher internal consistency among 43 subcategories of altered PECS.

\section{Discussion}

The chief objective of the existing study was to measure the reliability and validity of altered and modified PECS for autistic children of Pakistan. Psychometric calculation and improvement of social norms are involved for the consistency of every test. In the last stage of final trial the consistency of PECSPAK ${ }_{\text {was accomplished }}$ and it has concluded by the post testing with the altered PECS on the sample of seventy (70) normal and thirty (30) autistic children to validate the tool. Detailed calculation of comprehensive altered in other words tailored and modified pictures was approved, through statistics techniques. The main task of these techniques was to advance the reliability of the tool. Psychometric calculation and valuation of intrusion tools is obligatory to implement the tool in any applied field. The appropriate norms were established for the Pakistani community by the use of appropriate evaluation methods. Taking into consideration, the connection of male and female, related to a substantial gender-based population don't seem to comprehend any significant pressure on the responses of children. And it is reliable with the conclusions of the study on altered and modified PECS Weiss et al. (2006). 
while any precise and detailed disparity is not recognized in the presentations of people between both genders, i.e., male and female. Moreover, it's irrelevant effect of gender might be the reason for such modifications. The chief reason for such type of differences is that in this study we took the sample from a section where all the girls and boys got the same educational and learning chances. Their schools have the equivalent standards and set designs; their syllabus is also related to their capability, expertise, knowledge and intellectual skills are also the same due to the similar situation.

So, it has been determined that these conclusions and exploration indorses that there is dire need to do hard work for modifying and for the standardization of altered PECS in Pakistan. The current study is considered as the first step; it is not the end, so appraisals regarding its community-based relevancy and psychometric prospective are highly suggested. In another word it's the pioneer effort in this region to provide this tool with alteration and its modification to rehabilitate the autistic population.

Table 1 displayed total reliability coefficient centred on autistic student's recognition for 43 categories of altered PECS during post-testing phase. The total alpha coefficient for 43 items was in good range as its value 0.868 was greater than 0.8 . Greater than 0.8 value of alpha established relatively higher internal consistency among 43 subcategories of altered PECS. Hence altered PECS was evidenced to be a reliable tool for the sample of autistic children of Pakistan. Table 2 indicated total reliability coefficient centred on normal student's recognition for 43 categories of altered PECS during post-testing phase. The total alpha coefficient for 43 items was in good range as its value 0.8369 was greater than 0.8 . Greater than 0.8 value of alpha determined relatively higher internal consistency among 43 subcategories of altered PECS. Hence altered and modified PECS was evidenced to be a reliable tool for the sample of Pakistan. At last, all the modified/altered and translated material was presented to the experts for correct preparation and procedures. They revised all the contents in detail. The committee suggested minor developments in the wording and the preparations of pictures. It is reliable and valid tool and measured and evaluated by the internal consistency, inter-rater and test-retest reliability.

Furthermore altered PECS has also met all validity types except convergent validity. Altered PECS met all the criteria of content validity including concurrent validity, predictive/discriminant, cross-cultural validity and construct validity.

The existing research presents a number of procedures and measures to be assimilated in the alteration procedure, in adding together to the statistical investigation. Itemize these aspects, predominantly statistical measures, is outside the extent of this piece of writing; on the other hand, our directorial principles, the course of actions and references might provide as a foundation for the investigator to look for supplementary improvements in the subject matter of the alteration of the psychological tool. Lastly summing up the alteration of PECS could evidence to be a 
useful and well-organized tool for the development of practical/functional communication of autistic children in the region.

\section{Limitations and Future Recommendations}

Transmission of this study into the twin cities (Rawalpindi \& Islamabad) as to get the sample of conversable autistic and normal children of age group 5-10 years old both boys and girls is the major limitation of the study.

On the basis of results and conclusions, following recommendations are proposed:

- As PECSPAK is appropriate for the Pakistani culture and it advances the communication aptitudes of autistic children, it should be used as a teaching tool and instructional technology.

- In order to make applied application, teachers and professionals will be able to use with expertise, many of the influential instruments, re-design their educational plans through the practically oriented resources. Moreover they will be able to resolve the real problems of students having special needs during classroom teaching, specifically autistic children. Parents, teachers, audiologists, psychologists, occupational and vocational therapists and teachers should all work as a team in order to develop the applied interaction skills in autistic children.

\section{Implications}

Transmission of this study is the pioneer effort in the whole Asian Region with the aim to provide altered, translated and standardized version of this extraordinary tool to rehabilitate the autistic children. A tailored and standardized PECSPAK, which have been used for many children of different ages, is the outcome of current research. Due to outstanding results, clarity and its clinical implication, PECS is widely used tool for the autistic population as well as normal all over the world all along through its various altered and translations. Mainly across the societies and proportional studies, PECSPAK could be able to utilize as a constant and appropriate tool for data or information collection.

\section{Conclusion}

This study designates that PECS PAK is an operative tool, suitable for the culture of Pakistan. In the last stage of this study the consistency of PECSPAK was accomplished and it has finalized by the post testing with the altered PECS on the 
sample of seventy (70) normal and thirty (30) autistic children to validate the tool. Detailed calculation of complete altered in other words tailored pictures was accepted, through statistics techniques. The purpose of these techniques was to improve reliability of the tool. Psychometric calculation and valuation of feedback of intervention tools is compulsory to implement the tool in any practical field.

- The reliability of altered PECS was estimated by internal consistency, interrater, and test-retest reliability. The results illustrates that altered PECS has high test-retest reliability, and the mean reliability coefficients of altered PECS are higher than original PECS for the sample of Pakistan which proves that adapted PECS has sound reliability.

- Altered PECS has also met all validity types except convergent validity. It met all the criteria of content validity including concurrent validity, predictive/discriminant, cross-cultural validity and construct validity. 


\section{References}

Beaton, D. E., Bombardier, C., Guillemin, F., \& Ferraz, M. B. (2000). Guidelines for the process of cross-cultural adaptation of self-report measures. Spine, 25(24), 3186-3191. Lippincott Williams \& Wilkins, Inc.

Bondy, A., \& Frost, L. (2001). The picture exchange communication system. Behavior Modification, 25, 725 744. Retrieved from https://www.scirp.org/(S(i43d yn45teexjx455qlt3d2q))/reference/ReferencesPapers.aspx?ReferenceID=1763701

Cassepp-Borges, V., Baslbinotti, M. A., \&Teodoro, M. L. (2010). Tradução e validação de conteúdo: umaproposta para aadaptação de instrumentos. InstrumentaçãoPsicológica: Fundamentos E Práticas, (pp. 506520).New York, NY: Basic Books.

Frost, L., \&Bondy, A. (2012). The picture exchange communication system training manual (2nded.). Delware, USA: Pyramid Educational Consultants, Inc., 13 Garfield Way, Newark, DE.

International Test Commission. (2010). International Test Commission guidelines for translating and adapting tests. Retrieved from https://www.tandfonline.com/doi/abs/10.1080/15305058.2015.1111221

Kumpfer, K. L., Alvarado, R., Smith, P., \& Bellamy, N. (2002). Cultural sensitivity and adaptation in family-based prevention interventions. Prevention Science, 3(3), 241-246. Retrieved from https://www.researchgate.net/publication/11073510_

Sireci, S. G., Yang, Y., Harter, J., \& Ehrlich, E. J. (2006). Evaluating guidelines for test adaptations: A methodological analysis of translation quality. Journal of CrossCultural Psychology, 37(5), 557-567.

Tincani, M., \&Devis, K. (2011). Quantitative synthesis and component analysis of single-participant studies on the picture exchange communication system. Journal of Remedial and Special Education, 32(6), 458-470.

Weiss, M. G., Ramakrishna, J., \& Somma, D. (2006). Health-related stigma: rethinking concepts and interventions. Journal of Psychology, Health $\mathcal{E}$ Medicine, 11(3), 277-287. 